\section{Novas opções terapêuticas na síndrome de aspiração de mecônio}

\author{
New therapeutic options in meconium \\ aspiration syndrome
}

\author{
Mauricio Obal Colvero 1 \\ Aline Pieruccini Colvero 2 \\ Renato Machado Fiori 3 \\ Pedro Celiny Ramos Garcia 4
}

1-4 Disciplina de Terapia Intensiva Pediátrica. Pós-Graduação em Medicina/Pediatria e Saúde da Criança. Faculdade de Medicina da Pontifícia Universidade Católica do Rio Grande do Sul. Av. Ipiranga, 6690 - $5^{\circ}$ andar . Porto Alegre, RS, Brasil. CEP: 90.610-000. E-mail: celiny@terra.com.br

\begin{abstract}
Objectives: to review the literature on meconium aspiration syndrome (MAS) focusing on clinical aspects, pathophysiology, and treatment with emphasis on surfactant and bronchoalveolar lavage.

Methods: review including articles from MEDLINE, SCIELO and abstracts published in the national and international literature, from 1988 to 2004 using the keywords meconium aspiration syndrome, surfactant and bronchoalveolar lavage. Randomized and quasi-randomized trials, case control studies, meta-analyses and recently published reviews were selected. Other articles were included for their valuable contribution to the subject.

Results: the discovery of new pathophysiological mechanisms ensued new therapeutic options availability. MAS management is initiated with airway aspiration in the delivery room, followed by the ventilatory management required to maintain optimal arterial oxygenation, as well as complications treatment. Considering evidences showing that meconium mechanical airway obstruction and its inhibitory effect on the surfactant system, the use of surfactant replacement and bronchoalveolar lavage with surfactant suspension are under study.

Conclusions: experimental studies and studies focused on newborn using different surfactant suspensions have demonstrated controversial results. Therefore, it is very important to identify new ventilatory strategies and evaluate whether there are advantages in using surfactant and bronchoalveolar lavage with surfactant suspension in MAS.
\end{abstract}

Key words Meconium aspiration syndrome, Pulmonary surfactants, Bronchoalveolar lavage

\section{Resumo}

Objetivos: revisar a literatura sobre a síndrome de aspiração de mecônio (SAM), enfocando aspectos clínicos, fisiopatológicos e abordagem terapêutica, com destaque ao uso do surfactante e lavado broncoalveolar.

Métodos: revisão baseada em artigos publicados na MEDLINE, SCIELO e resumos de congressos internacionais de 1988 a 2004, incluindo ensaios randomizados ou quasi-randomizados, estudos caso-controle $e$ metanálises.

Resultados: devido à comprovação da inibição do surfactante na SAM, houve modificações em sua abordagem terapêutica. O manejo atual consiste na aspiração das vias aéreas na sala de parto, seguida de suporte ventilatório necessário para manter a oxigenação arterial adequada, e tratamento das complicações. Tendo em vista a obstrução mecânica do mecônio e seu efeito inibitório sobre o surfactante, a reposição e lavado broncoalveolar com surfactante estão sendo estudados atualmente.

Conclusões: estudos em animais e em recémnascidos apresentam resultados controversos quanto aos benefícios do uso de surfactante e lavado broncoalveolar na SAM. Torna-se importante a realização de mais estudos para avaliar novas estratégias ventilatórias e se existem vantagens no uso do surfactante e lavado broncoalveolar com surfactante na SAM.

Palavras-chave Síndrome de Aspiração de Mecônio, Surfactantes Pulmonares, Lavagem Broncoalveolar 


\section{Introdução}

A Síndrome de Aspiração de Mecônio (SAM) é uma importante causa de morbi-mortalidade no período neonatal e caracteriza-se por graus variados de insuficiência respiratória. ${ }^{1} \mathrm{O}$ mecônio está presente no líquido amniótico em cerca de 10 a $15 \%$ dos partos, ${ }^{2-}$ 4 sendo que cerca de $5 \%$ dos recém-nascidos com líquido amniótico meconial desenvolverão SAM. ${ }^{3}$ Destes, 25 à $60 \%$ necessitam de ventilação mecânica, dos quais 3 à $12 \%$ não respondem à terapêutica instituída e evoluem para óbito.5,6 A SAM grave é uma das principais indicações de uso de membrana de oxigenação extra-corpórea "extracorporeal membrane oxygenation" (ECMO), 1,3 perfazendo cerca de $35 \%$ das indicações do procedimento. A ECMO é extremamente onerosa, invasiva e não está disponível em nosso meio. A presença do mecônio no líquido amniótico é mais comum quanto maior a idade gestacional e em recém-nascidos pequenos para a idade gestacional. 7 Nas gestações com mais de 42 semanas, ocorre em aproximadamente $30 \%$ dos partos. 4

Mesmo após a aspiração das vias aéreas pelo obstetra e pelo pediatra, alguns bebês que nascem com líquido amniótico meconial desenvolvem SAM. ${ }^{3}$ Seu tratamento é inespecífico 6 e consiste no suporte ventilatório necessário e manejo das complicações. Tendo em vista a obstrução mecânica do mecônio e seu efeito inibitório sobre o surfactante, 8 a reposição e lavado broncoalveolar com surfactante têm mostrado benefícios e vêm sendo estudados nos últimos anos.

Nesta revisão foram considerados artigos das bases MEDLINE, SCIELO e resumos publicados em congressos internacionais, entre 1984 e 2004, utilizando-se as palavras-chave síndrome de aspiração de mecônio, surfactante e lavado broncoalveolar. Selecionamos ensaios randomizados ou quasi-randomizados, estudos de caso-controle, metanálises e artigos de revisão recentemente publicados. Alguns outros artigos foram selecionados devido à importância para o tema.

\section{Apresentação clínica e laboratorial}

A SAM pode se apresentar desde uma disfunção respiratória leve até um quadro grave que evolui para a morte, apesar do manejo clínico adequado. ${ }^{4} \mathrm{~A}$ SAM tipicamente se apresenta como disfunção respiratória, taquipnéia, gemência, batimento de asas do nariz, tempo expiratório prolongado e hipoxemia logo após o nascimento em um recém-nascido com história de mecônio espesso no líquido amniótico, impregnação por mecônio nas unhas, cabelos e cordão umbilical, ou ainda presença de mecônio espesso abaixo das cordas vocais na aspiração traqueal sob visualização direta. ${ }^{4}$ Pode apresentar características somáticas de pós-maturidade e manifestações variadas de asfixia perinatal. O aumento do diâmetro ântero-posterior do tórax ou tórax em barril, devido à doença obstrutiva das vias aéreas, é comum na SAM. A ausculta pulmonar é inespecífica e podem ser audíveis estertores difusos e diminuição de murmúrio devido a atelectasia ou pneumotórax. Entre as complicações mais freqüentes e graves destacam-se o pneumotórax e a hipertensão pulmonar persistente do recém-nascido, que é observada em mais da metade dos pacientes com SAM grave. 4 Além disso, achados diversos relacionados à asfixia podem ser encontrados, entre eles a hipotensão, coagulação intravascular disseminada, acidose metabólica, anemia, insuficiência cardíaca e crises convulsivas. As alterações metabólicas encontradas pode ser decorrência da asfixia, tais como hipoglicemia e hipocalcemia.

Radiologicamente, a SAM se apresenta com infiltrados grosseiros, áreas de consolidação com hiperinsuflação e, em $30 \%$ dos casos, pode haver derrame pleural. Há um risco aumentado de pneumotórax e pneumomediastino, acometendo aproximadamente $25 \%$ dos pacientes gravemente enfermos. As radiografias de tórax são alteradas em mais de metade dos recém-nascidos com mecônio abaixo das cordas vocais, mas menos de $50 \%$ dos pacientes com alterações radiológicas apresentam disfunção respiratória significativa. A gravidade das alterações radiológicas pode não estar correlacionada com a gravidade da doença clínica. 4

Outros exames necessários incluem gasometrias seriadas, hemograma, culturas e pesquisa de Listeria monocitogenes, especialmente nos recém-nascidos com idade gestacional abaixo de 34 semanas.

\section{Fisiopatologia}

O mecônio aparece primeiramente no íleo fetal entre a décima e a décima-sexta semanas de gestação como um líquido viscoso e esverdeado composto por secreções gastrointestinais, restos celulares, sucos gástrico e pancreático, muco, sangue, lanugo e vérnix. O mecônio é composto em aproximadamente 72 a $80 \%$ de água. A composição do seu peso seco consiste em mucopolissacarídeos e, em menor quantidade, em proteínas e lipídios. 4 Apesar do mecônio intestinal aparecer precocemente no intestino fetal, a 
presença de mecônio no líquido amniótico raramente ocorre antes das 38 semanas de gestação. ${ }^{4} \mathrm{~A}$ associação entre líquido amniótico meconial e sofrimento fetal é bastante controversa; para alguns autores a eliminação do mecônio pelo feto ainda na cavidade uterina ocorre devido a um aumento na peristalse intestinal secundário à hipoxemia e sofrimento fetal. Para outros, a compressão abdominal durante o trabalho de parto e o reflexo vagal secundário à compressão do pólo cefálico explicariam a presença do mecônio no líquido amniótico, sem necessariamente representar sofrimento fetal. ${ }^{5}$ Os antecedentes maternos mais freqüentemente observados são a hipertensão arterial, doença cardiovascular ou pulmonar crônica, hipotensão aguda, descolamento prematuro de placenta, placenta prévia, partos laboriosos, presença de circulares, prolapsos e nós de cordão e apresentação pélvica.

Nos partos em que se observa a presença de mecônio no líquido amniótico, os primeiros movimentos respiratórios do recém-nascido fazem com que o mecônio migre das vias aéreas centrais para a periferia dos pulmões. 9 Devido ao poder de aderência do mecônio e à pouca eficácia do sistema mucociliar em expelí-lo das vias aéreas, o mecônio pode depositar-se com muita facilidade. 1

A fisiopatologia da SAM está relacionada à obstrução de pequenas vias aéreas por partículas de mecônio, levando a múltiplas áreas de atelectasia pulmonar, seguida de pneumonite química mediada por leucócitos, enzimas e interleucinas e, possivelmente, a ocorrência de infecção bacteriana secundária. 3,10 Como conseqüência desse processo, podem ocorrer graus variados de edema intersticial, vasoconstrição arterial pulmonar, redução da complacência pulmonar, seguida de hipertensão pulmonar persistente e insuficiência respiratória grave. 5

O mecônio pode ocasionar uma obstrução parcial ou total das vias aéreas inferiores. A obstrução parcial da via aérea permite a entrada do ar, mas não a sua saída do alvéolo. Esse mecanismo de válvula é responsável pelo alçaponamento progressivo de ar no pulmão, aumentando o risco de pneumotórax.9,11,12 Por outro lado, a obstrução completa de pequenas vias aéreas pode resultar em atelectasias regionais e alteração da ventilaçãoperfusão, levando à hipoxemia. ${ }^{4}$

Além de seus efeitos obstrutivos, estudos evidenciam que a inativação do surfactante pelo mecônio também tem papel importante na disfunção pulmonar encontrada na SAM,8,13-15 levando a atelectasia e alteração da relação ventilação/perfusão (V/Q).12 Vários dos componentes do mecônio inibem a função do surfactante, porém os mais estu- dados, in vivo 16 e in vitro, 8 são os ácidos graxos livres. Seu efeito inibitório parece ser dose-dependente. ${ }^{5}$ Luz et al. ${ }^{14}$ demonstraram a inibição do surfatante pelo mecônio in vitro através do uso do teste das microbolhas estáveis. ${ }^{14} \mathrm{~A}$ instilação de mecônio em pulmões caninos levou a uma diminuição da complacência pulmonar, da capacidade residual funcional e da oxigenação, 15 corroborando a hipótese da inibição do surfactante. Além do efeito inibitório direto do mecônio sobre o surfactante, a hiperinsuflação pulmonar pelo alçaponamento de ar, associado à lesão alveolar pela ventilação mecânica, causam um extravasamento de conteúdo protéico no alvéolo, inibindo ainda mais a função do surfactante. 12

\section{Prevenção}

O acompanhamento pré-natal, o cálculo preciso da idade gestacional e a monitorização do bem-estar fetal no decorrer do trabalho de parto são fatores fundamentais na prevenção da SAM. Alterações no perfil biofísico fetal, desacelerações tardias e acidose fetal são indicações para intervenção cirúrgica imediata. Antes do final da década de 70, pensava-se que a aspiração do líquido amniótico meconial ocorria nos primeiros movimentos respiratórios após o nascimento. Era realizada a aspiração da nasofaringe antes do desprendimento dos ombros e antes do primeiro movimento respiratório. A traquéia era imediatamente entubada e aspirada para limitar a aspiração do mecônio da orofaringe e da traquéia, um procedimento que permanece como rotina em muitas instituições. A SAM continua ocorrendo em pacientes adequadamente aspirados na sala de parto. 4

O uso da aspiração traqueal direta em todos os pacientes com líquido amniótico meconial está sob reavaliação. Atualmente, é proposto que todos os recém-nascidos expostos ao mecônio tenham a orofaringe e a nasofaringe aspirada com sistema de sucção de parede, e a entubação traqueal imediata com aspiração é recomendada apenas em pacientes com depressão ao nascer (previsão de Apgar do primeiro minuto menor que sete). Esse tipo de protocolo reduziu a entubação de emergência em $40 \%$ sem aumentar a incidência ou gravidade dos casos de SAM. ${ }^{4}$ A ventilação com pressão positiva somente deve ser realizada após a remoção do mecônio presente nas vias aéreas e preconiza-se a realização de aspirado gástrico para diminuir o risco de vômitos e conseqüente aspiração. ${ }^{7}$ Não tem sido utilizada a instilação endotraqueal de soro fisio- 
lógico, uma vez que tal procedimento parece ser ineficaz e pode desencadear uma piora da mecânica pulmonar. 5

A amnioinfusão, que consiste na infusão de solução salina aquecida na cavidade amniótica, foi descrita como muito útil em pacientes com trabalho de parto complicado, por passagem de mecônio espesso, melhorando a avaliação do escore de Apgar ao nascer, reduzindo a quantidade de mecônio abaixo das cordas vocais e também diminuindo a necessidade de ventilação com pressão positiva e melhorando o pH sangüíneo do cordão umbilical. ${ }^{17}$ As finalidades da infusão de líquido são: corrigir uma eventual oligodramnia, reduzir o risco de compressão do cordão umbilical e diluir o mecônio. 5 Apesar dos resultados indicarem algum benefício, o risco de intercorrências obstétricas, tais como amnionite, pode chegar a $35 \%, 5$ restringindo seu uso.

\section{Tratamento}

Todo recém-nascido com história de líquido amniótico meconial e desconforto respiratório deve ser observado e monitorizado. Controle térmico, correção de anemia, manutenção da pressão arterial, infusão de glicose e homeostase hidroeletrolítica são fundamentais no manejo do recém-nascido com SAM. O uso de antibioticoterapia é controverso, mas costuma ser recomendado devido à gravidade do quadro e dificuldade de exclusão de processo infeccioso. O mecônio apresenta grande potencial de colonização por germes, especialmente gram negativos.4,5 A indicação de fisioterapia respiratória deve ser individualizada de acordo com o grau de labilidade de oxigenação e risco de pneumotórax, havendo indicação apenas nos pacientes mais estáveis. 5

A saturação deve ser mantida acima de $92-95 \%$, idealmente avaliada pré e pós-ductal para o diagnóstico de hipertensão pulmonar persistente. $\mathrm{O}$ analisador transcutâneo de $\mathrm{PCO}_{2}$ deve ser usado quando disponível. Considera-se o uso de ventilação mecânica e cateter arterial umbilical quando a fração inspirada de oxigênio ultrapassar $60 \%$. A hipoxemia, hipercapnia e acidose metabólica são os achados gasométricos mais comuns. O tratamento da SAM é baseado no suporte ventilatório necessário para manter a oxigenação arterial adequada $(\mathrm{PaO} 2$ entre 50 e $70 \mathrm{mmHg}$ ), $\mathrm{pH}$ acima de 7,20 e $\mathrm{PaCO}_{2}$ entre 40 e $60 \mathrm{mmHg}$ e no tratamento das complicações, bem como o manejo de sequielas da asfixia perinatal.

Têm sido descritas diversas modalidades de ventilação mecânica na SAM.18-22 A pressão positiva contínua nas vias aéreas "continuous positive airway pressure" (CPAP) com pressões baixas (1-3 $\left.\mathrm{cmH}_{2} \mathrm{O}\right)$ e intermediárias $\left(4-7 \mathrm{cmH}_{2} \mathrm{O}\right)$ é descrita como benéfica, provavelmente através da resolução de atelectasias e estabilização de vias aéreas terminais em colapso, 18 mas deve ser utilizada com cautela na hipertensão pulmonar do recémnascido, 19 e ainda pode agravar a hiperinsuflação pulmonar. ${ }^{4} \mathrm{O}$ uso do CPAP deve ser considerado nas doenças mais leves, com monitorização cuidadosa de complicações. ${ }^{5}$ A ventilação convencional está indicada nos casos mais graves ou na falha do uso do CPAP nasal e sua aplicação não deve ser retardada para não piorar um quadro de hipertensão pulmonar. O manejo do ventilador na SAM é bastante difícil, tendo em vista o comprometimento heterogêneo do parênquima pulmonar, com áreas de padrão obstrutivo e restritivo alternando com áreas de pulmão normal.20 Utilizam-se ventiladores de fluxo contínuo, limitados por pressão e ciclados a tempo, com parâmetros mínimos necessários para manter uma oxigenação e ventilação adequadas. A pressão inspiratória deve ser suficiente para promover a expansão da caixa torácica, em geral em torno de 20 a $30 \mathrm{cmH}_{2} \mathrm{O}$. Pressões expiratórias altas, principalmente associadas à frequiência respiratória elevada, podem ocasionar a pressão positiva no final da expiração "positive end-expiratory pressure" (PEEP) inadvertente, aumentando o risco de alçaponamento de ar. Um tempo inspiratório de 0,3 a 0,4 segundos é recomendado e deve ser associado a uma freqüência respiratória acima de $40 \mathrm{mrpm}$ para ventilar porções de pulmão normal sem comprometer as áreas com obstrução parcial. O volume corrente, quando o respirador permite sua aferição, deve ser mantido entre 5 e $7 \mathrm{ml} / \mathrm{kg}$ e o fluxo em torno de $8 \mathrm{~L} / \mathrm{min}$ para gerar uma curva de pressão-volume sem platô na monitorização gráfica. As modificações na assistência ventilatória devem ser avaliadas através de controle gasométrico.

Outras estratégias ventilatórias têm sido estudadas. A ventilação mecânica sincronizada, "synchronized intermittent mandatory ventilation" (SIMV), na qual o ciclo do respirador é desencadeado em sincronia com o movimento inspiratório do recém-nascido, não tem evidências de vantagens quando utilizada em paciente com SAM. ${ }^{5}$ A ventilação mecânica de alta freqüência tem sido utilizada na SAM, assim como na doença das membranas hialinas como estratégia de resgate quando a ventilação convencional não é eficaz, mas os relatos sobre a segurança e eficácia são conflitantes. 18 A ventilação líquida com perfluorocarbono tem sido estu- 
dada em síndromes aspirativas, como a SAM, com a intenção de remover o mecônio, promover o recrutamento alveolar, reduzir a lesão pulmonar causada pelos ventiladores e melhorar a relação ventilação/perfusão.21,22 Seu uso precoce reduziria a inativação do surfatante e o processo inflamatório. Existem estudos em andamento para avaliar sua eficácia em diferentes doenças respiratórias que acometem recém-nascidos, incluindo a SAM. ${ }^{22}$

A membrana de oxigenação extracorpórea (ECMO) tem indicação quando ocorre falha no manejo ventilatório com as diversas estratégias de ventilação. É um tratamento dispendioso e envolve uma infra-estrutura muito grande, limitando seu uso em nosso meio. Em outros centros, têm sido observada uma redução da mortalidade nos casos de SAM complicada com hipertensão pulmonar persistente. 5

\section{Surfactante e lavado broncoalveolar}

A reposição do surfactante na doença da membrana hialina está bem estabelecida através de diversos ensaios clínicos randomizados, multicêntricos e controlados realizados entre recém-nascidos prematuros. Comprovadamente, o uso do surfactante melhora a oxigenação, reduz a necessidade de ventilação mecânica e tempo de internação, além de diminuir a incidência de complicações respiratórias e mortalidade entre as crianças. Em recém-nascidos a termo com insuficiência respiratória, o papel do surfactante não está tão bem definido. Diversas doenças que acometem recém-nascidos a termo cursam com deficiência ou inibição do surfactante, entre elas a SAM. Tendo em vista a obstrução mecânica do mecônio e seu efeito inibitório sobre o surfactante, 8 a reposição e o uso de lavado broncoalveolar com surfactante estão sendo estudados atualmente.

O uso do surfatante exógeno tem como base a reversão da disfunção do surfatante pulmonar e um possível efeito detergente sobre o mecônio. Os ácidos graxos presentes no mecônio são os principais inibidores da função do surfactante. 16 Entretanto, se em grandes concentrações, o surfactante pode reverter o efeito inibitório do mecônio. ${ }^{1}$ Baseando-se nessa hipótese, Auten et al.23 realizaram um ensaio clínico não controlado com sete recém-nascidos a termo em ventilação mecânica por SAM. Foi utilizado um surfactante bovino em até quatro doses de $90 \mathrm{mg} / \mathrm{kg}$ melhorando o gradiente alvéolo-arterial de oxigênio e índice de oxigenação, pelo menos a curto prazo. Nenhum paciente morreu e não houve complicações no procedimento, motivando o surgimento de novos estudos controlados. Khammash et al. ${ }^{24}$ analisaram retrospectivamente os dados de 20 recém-nascidos a termo em ventilação mecânica com SAM tratados com uma a três doses de surfactante bovino em doses de 100 $\mathrm{mg} / \mathrm{kg}$. Foi observada melhora no gradiente alvéoloarterial de oxigênio e índice de oxigenação. Findlay et al.$^{9}$ realizaram um ensaio clínico randomizado e controlado com placebo envolvendo 40 recémnascidos com SAM grave. Nesse estudo, o surfactante (Survanta) foi administrado precocemente no quadro de insuficiência respiratória, com três doses de $150 \mathrm{mg} / \mathrm{kg}$ a cada seis horas. Foram observadas melhora na oxigenação arterial, diminuição de escape de ar, redução na necessidade de ECMO, duração da ventilação mecânica e necessidade de oxigênio quando comparado ao placebo. A resposta foi mais evidente após a segunda e terceira doses. Os autores sugeriram que a resposta parcial à primeira dose ocorreu devido à inativação do surfactante pelo mecônio presente na via aérea. As doses subseqüentes superariam essa inibição e ficariam depositadas na superfície alveolar, restabelecendo a superfície ar-líquido e melhorando a oxigenação. Lotze $e t$ al. 25 demonstraram que o surfactante administrado reduziu a duração da ECMO para os recém-nascidos. Outro grande ensaio clínico randomizado multicêntrico envolvendo 44 centros foi realizado por Lotze et al.,26 demonstrando que o uso de surfactante (Survanta) administrado até o quinto dia de vida na insuficiência respiratória em recém-nascidos a termo diminuiu significativamente a necessidade de uso da ECMO. Essa redução foi mais evidente em recémnascidos com insuficiência respiratória por SAM e índice de oxigenação menor (entre 15 e 22). Nesse estudo, 328 recém-nascidos a termo com SAM e hipertensão pulmonar foram randomicamente alocados a receber quatro doses de Survanta (100 $\mathrm{mg} / \mathrm{kg}$ ) a cada seis horas ou placebo com ar, havendo uma redução de $41 \%$ na indicação de ECMO no grupo que utilizou surfactante quando comparado com o grupo placebo. Tendo em vista que a ECMO não está disponível na maioria das unidades neonatais em nosso meio e é indicada em casos refratários ao tratamento convencional, uma redução de indicação de ECMO possivelmente significaria redução em mortalidade. O uso do lavado broncoalveolar com soro fisiológico isoladamente tem resultados conflitantes, com piora do desconforto respiratório em recém-nascidos 27 e com tendência a melhora da oxigenação arterial quando realizado em modelos experimentais de SAM em $\operatorname{porcos} 28$ e coelhos. $29 \mathrm{Um}$ estudo experimental realizado por Marraro et al. 30 demonstrou piora significativa da 
função pulmonar com o uso de lavado broncoalveolar com soro fisiológico, não recomendando seu uso. $\mathrm{O}$ autor sugere que parte do soro fisiológico administrado no lavado possa ser prontamente absorvido no alvéolo, piorando as trocas gasosas, e inativando o surfactante.

O uso do lavado broncoalveolar com surfactante visa remover as substâncias nocivas presentes na via aérea dos recém-nascidos com SAM, entre elas o próprio mecônio, neutrófilos, exsudato proteico e mediadores inflamatórios. ${ }^{6} \mathrm{O}$ surfactante também exerce um efeito detergente diminuindo a aderência do mecônio. Além desses efeitos mecânicos, sugerese que parte do surfactante administrado no lavado poderia permanecer no alvéolo pulmonar, diminuindo a tensão superficial e melhorando as trocas gasosas. 1

Paranka et al.28 foram os primeiros autores a realizar lavado broncoalveolar com surfactante em modelos experimentais de aspiração de mecônio em porcos. Ficou demonstrado que a lavagem com surfactante é mais efetiva que a realizada com soro fisiológico e melhora a função pulmonar e a oxigenação arterial. Balaraman et al. 31 evidenciaram que o surfactante diluído e administrado através de lavagem broncoalveolar em modelos experimentais de SAM tem uma melhor distribuição no parênquima e até um terço da solução com surfactante permaneceu no pulmão. O mesmo autor comparou a administração de três diferentes soluções de surfactante em lavado broncoalveolar (infrasurf, surfactante KL4 e exosurf) em concentrações de 4 a 4,5 $\mathrm{mg} / \mathrm{ml}$ do fosfolipídio em porcos com SAM experimental. Todas as soluções foram efetivas na reversão da injúria pulmonar. 32 Ohama et al.33 demonstraram que o lavado broncoalveolar com uma solução de surfactante, em coelhos com SAM induzida, foi eficaz em remover o mecônio da via aérea, e melhorou a oxigenação e ventilação dos modelos. Cochrane et al. ${ }^{29}$ realizaram três lavados broncoalveolares com surfaxin em cada cobaia com modelo experimental de SAM. Houve melhora substancial da oxigenação arterial e padrão radiológico no grupo tratado em comparação com o controle. Além da melhora dos parâmetros clínicos, a comparação dos achados histológicos mostrou melhor expansão pulmonar e menos depósito de exsudato proteico no grupo que utilizou surfactante, em comparação com um grupo controle e outro que realizou lavado apenas com soro fisiológico. Outro estudo experimental com um grande número de animais com SAM não evidenciou melhora significativa no grupo que recebeu surfactante. $34 \mathrm{Em} \mathrm{um}$ estudo piloto em humanos para avaliar a eficácia e segurança do lavado broncoalveolar com surfactante, Lam et al. ${ }^{1}$ realizaram lavado broncoalveolar com $15 \mathrm{ml} / \mathrm{kg}$ de surfactante diluído em solução salina a uma concentração de fosfolipídio de $5 \mathrm{mg} / \mathrm{ml}$ nas primeiras seis horas de vida, em 6 recém-nascidos com SAM severa, sendo comparados com controles históricos. Em 48 horas, foi observada melhora do índice de oxigenação, diminuição da pressão média de via aérea, diminuição da necessidade de oxigênio e melhora do gradiente alvéolo-arterial de oxigênio. Também foram observadas reduções no tempo de ventilação mecânica e uso de oxigênio no grupo tratado com surfactante. $\mathrm{O}$ procedimento foi bem tolerado e não teve complicações.

Recentemente, Wiswell et al. ${ }^{6}$ realizaram um estudo piloto aberto, multicêntrico, randomizado e controlado avaliando o efeito do lavado broncoalveolar com um surfactante que contém um peptídeo similar à proteína B (lucinactante) na SAM. Foram incluídos pacientes com mais de 35 semanas de idade gestacional, até o terceiro dia de vida, em ventilação mecânica com índice de oxigenação entre 8 e 25 . Vinte e dois pacientes foram randomizados a receber o tratamento convencional para SAM, sem surfactante, ou lavado broncoalveolar com solução de surfactante a $2,5 \mathrm{mg} / \mathrm{ml}$ realizado em volumes de $8 \mathrm{ml} / \mathrm{kg}$ por duas vezes. O terceiro lavado foi realizado com uma solução de $10 \mathrm{mg} / \mathrm{ml}$ de fosfolipídio. Cerca de $50 \%$ da solução instilada foi recuperada com a aspiração traqueal, sendo mais efetiva quando os recém-nascidos estavam sedados. O procedimento foi considerado seguro e no grupo dos recém-nascidos em que foi utilizado o lavado broncoalveolar houve uma tendência para serem retirados mais precocemente da ventilação mecânica e diminuição do índice de oxigenação nas primeiras 96 horas. O acompanhamento realizado no primeiro ano de vida não mostrou diferença entre os grupos em relação à necessidade de reinternações, mortalidade e desenvolvimento.

Em um relato de casos de SAM com hemorragia pulmonar, Kaneko et al. ${ }^{35}$ utilizaram uma pequena dose complementar de surfactante $(40 \mathrm{mg} / \mathrm{kg}$ ) após o lavado broncoalveolar com surfactante diluído, observando melhora na oxigenação e desmame da ventilação mecânica. A utilização de lavado broncoalveolar com surfactante diluído seguido de uma dose complementar teria três propósitos: a) remoção do mecônio para desobstrução da via aérea; b) restabelecimento da função surfactante inibida pelo mecônio, e ainda c) reversão da disfunção do surfactante devido a um edema pulmonar secundário ao lavado broncoalveolar.3,35

A dose recomendada de surfactante atualmente é 
de 100 a $200 \mathrm{mg} / \mathrm{kg}$, instilado o mais precocemente possível em recém-nascidos com SAM grave, podendo ser repetida até duas à quatro doses em intervalos de 6 à 12 horas. 5 O lavado com surfactante diluído tem sido realizado com uma concentração de $5 \mathrm{mg} / \mathrm{ml}$, na dose de $15 \mathrm{ml} / \mathrm{kg}$ administrado nas primeiras seis horas de vida conforme descrito por Lam et al.. 1

Conforme visto anteriormente, diversos estudos em animais e estudos-piloto em humanos, utilizando diferentes estratégias para o uso do surfactante, foram desenvolvidos sugerindo reversão ou atenuação da doença pulmonar induzida pelo mecônio. A necessidade de utilizar altas doses de surfactante na maioria dos trabalhos provavelmente deve-se a distribuição não homogênea do surfactante na via aérea parcialmente obstruída e também devido à inativação do surfactante exógeno pelo mecônio. ${ }^{1}$ Devido aos resultados conflitantes com a utilização do surfactante e do lavado broncoalveolar com

\section{Referências}

1. Lam BCC, Yeung CY. Surfactant lavage for meconium aspiration syndrome: a pilot study. Pediatrics 1999; 103: 10148 .

2. Cleary GM, Wiswell TE. Meconium-stained amniotic fluid and the meconium aspiration syndrome - an update. Pediatr Clin North Am 1998; 45: 511-29.

3. Ohama Y, Ogawa Y. Treatment of meconium aspiration sindrome with surfactant lavage in an experimental rabbit model. Pediatr Pulmonol 1999; 28: 18-23.

4. Whitsett JA, Pryhuber GS, Rice WR, Warner BB, Wert SE. Acute respiratory disorders. In: Avery GB, Fletcher MA, MacDonald MG, editors. Neonatology: pathophysiology and management of the newborn. 5. ed. Philadelphia: Lippincott Williams \& Williams; 1999. p. 494-7.

5. Guinsburg R, Miyoshi MH. Síndrome da aspiração de mecônio. In: Alves Filho N, Trindade Filho O, organizadores. Clínica de perinatologia - aparelho respiratório em neonatologia. 1. ed. v. 1.São Paulo: Editora Médica e Científica; 2001. p. 273-90.

6. Wiswell TE, Knight GR, Finer NN, Donn SM, Desai H, Walsh WF et al. A multicenter, randomized, controlled trial comparing surfaxin (lucinactant) lavage with standard care for treatment of meconium aspiration syndrome. Pediatrics 2002; 109: 1081-7.

7. Diniz EMA, Ceccon MEJR. Síndrome de aspiração meconial. Pediatr Mod 2000; 36: 42-4.

8. Sun B, Curstedt T, Robertson B. Surfactant inhibition in experimental meconium aspiration syndrome. Acta Paediatr 1993; 82: 182-9.

9. Findlay RD, Taeusch HW, Walther FJ. Surfactant replacement therapy for meconium aspiration syndrome. Pediatr 1996; 97: 48-52. surfactante diluído na SAM, torna-se importante a realização de novos estudos para avaliar se existem vantagens no seu uso e definir a dose ideal e a forma de ser administrado.

\section{Prognóstico}

A mortalidade dos recém-nascidos com quadro de SAM grave pode variar de 10 a $60 \%$, sendo que o principal fator relacionado ao mau prognóstico é a presença de hipertensão pulmonar persistente. $5 \mathrm{~A}$ morbidade está relacionada com o grau de asfixia perinatal, seqüelas pulmonares e neurológicas. Os estudos com novas estratégias de tratamento, entre elas o uso do surfactante e lavado broncoalveolar com surfactante, evidenciam alguma melhora na oxigenação e redução de indicação de ECMO, entretanto, novos estudos são necessários para definir indicações precisas e impactos esperados com o uso de novas abordagens terapêuticas.

10. Tyler DC, Murphy J, Cheney FW. Mechanical and chemical damage to lung tissue caused by meconium aspiration. Pediatrics 1978; 62: 454-9.

11. Gooding CA, Gregory GA, Taker P. An experimental model for the study of meconium aspiration of the newborn. Radiology 1971; 100: 137-40.

12. Kattwinkel J. Surfactant lavage for meconium aspiration: a word of caution. Pediatrics 2002; 109: 1167-8.

13. Moses D, Holm BA, Spitale P, Lui M, Enhorning G. Inhibition of pulmonary surfactant function by meconium. Am J Obstet Gynecol 1991; 164: 477-81.

14. Luz JH, Fiori HH, Pereira MR, Piva J, Fiori RM. Stable microbubble test for evaluation of surfactant inhibition by meconium, human albumin and blood and restoration of surfactant activity by dextran. In: Annals of XVII International Workshop on Surfactant Replacement; 2002 May 24-26; Cagliary, Italy. Cagliary: Parma University; 2002. p. 16.

15. Davey AM, Becker JD, Davis JM. Meconium aspiration syndrome: physiological and inflammatory changes in a newborn piglet model. Pediatr Pulmonol 1993; 16: 101-8.

16. Clark DA. Nieman GF, Thompson JE, Paskanik AM, Rokhar JE, Bredemberg CE. Surfactant displacement by meconium free fatty acids: an alternative explanation for atelectasis in meconium aspiration syndrome. J Pediatr 1987; 110: 765-70.

17. Burchfield DJ. Physiology of resuscitation. In: Polin RA, Fox WW, editors. Fetal and neonatal physiology. 2. ed. Philadelphia: WB Sauders; 1998. p. 1028-9.

18. Calkovska A, Curstedt T, Renheim G, Robertson B. Combined effects of high-frequency ventilation and surfactant treatment in experimental meconium aspiration syndrome. Acta Anaesthesiol Scand 1999; 43: 135-45. 
19. Ahumada CA, Goldmith JP. Continuous distending pressure. In: Goldsmith JP, Karotkin EH, editors. Assisted ventilation of the neonate. 3. ed. Philadelphia: WB Sauders; 1996. p. 151-65.

20. Robinson TW, Roberts AM. Effects of exogenous surfactant on gas exchange and compliance in rabbits after meconium aspiration. Pediatr Pulmonol 2002; 33: 117-23.

21. Shaffer TH, Wolfson MR. Liquid ventilation. In: Polin RA, Fox WW, editors. Fetal and neonatal physiology. 2. ed. Philadelphia: WB Sauders; 1998. p. 1219-42.

22. Nakamura T, Matsuzawa S, Sugiura M, Tamura M. A randomised control study of partial liquid ventilation after airway lavage with exogenous surfactant in a meconium aspiration syndrome animal model. Arch Dis Child Fetal Neonatal Ed 2000;82: F160-2.

23. Auten RL, Notter RH, Kendig JW, Davis JM, Shapiro DL. Surfactant treatment of full-term newborn with respiratory failure. Pediatrics 1991; 87: 101-7.

24. Khammash H, Perlman M, Wojtulewicz J, Dunn M. Surfactant therapy in full-term neonates with severe respiratory failure. Pediatrics 1993; 92: 135-9.

25. Lotze A, Knight GR, Martin GR, Bulas DI, Hull WM, O'Donnell RM et al. Improved pulmonary outcome after exogenous surfactant therapy for respiratory failure in term infants requiring extracorporeal membrane oxygenation. J Pediatr 1993; 122: 261-8.

26. Lotze A; Mitchell BR; Bulas DI; Hull WM; O'Donnell RM; Whitsett JA; Short BL; Zola EM; Shalwitz RA; Gunkel JH. Multicenter study of surfactant (beractant) use in the treatment of term infants with severe respiratory failure. J Pediatr 1998; 132: 40-7.

27. Carson BS; Losey RW; Bowes WA; Simmons AS. Combined obstetric and pediatric approach to prevent meconium aspiration syndrome. Am J Obstet Gynecol 1976: $126: 712-5$.
28. Paranka MS; Walsh WF; Stancombe BB. Surfactant lavage in a piglet model of meconium aspiration syndrome. Pediatr Res 1992; 31: 625-8.

29. Cochrane CG, Revak SD, Merritt A, Schraufstätter IU, Hoch RC, Henderson C, Andersson S, Takamori H, Oades ZG. Bronchoalveolar lavage with KL4-surfactant in models of meconium aspiration syndrome. Pediatr Res 1998; 44: 705-15.

30. Marraro G, Bonati M, Ferrari A, Barzaghi MM, Pagani C, Bortolotti A et al. Perfluorocarbon broncho-alveolar lavage and liquid ventilation versus saline broncho-alveolar lavage in adult guinea pig experimental model of meconium inhalation. Intensive Care Med 1998; 24: 501-8.

31. Balaraman V, Sood SL, Finn KC, Hashiro G, Uyehara CFT, Easa D. Physiologic response and lung distribution of lavage versus bolus Exosurf in piglets with acute lung injury. Am J Respir Crit Care Med 1996; 153: 1838-43.

32. Balaraman V, Meister J, Ku TL. Lavage administration of dilute surfactants after acute lung injury in neonatal piglets. Am J Respir Crit Care Med 1998; 158: 12-7.

33. Ohama Y, Itakura Y, Koyama N, Eguchi H, Ogawa Y. Effect of surfactant lavage in a rabbit model of meconium aspiration syndrome. Acta Paediatr Jpn 1994; 36: 236-8.

34. Wiswell TE, Peabody SS, Davis JM, Slayter MV, Bent RC, Merrit TA. Surfactant therapy and high-frequency jet ventilation in the management of a piglet model of the meconium aspiration syndrome. Pediatr Res 1994; 36: 494-500.

35. Kaneko M, Watanabe J, Ueno E. Surfactant lavage and replacement in meconium aspiration syndrome with pulmonary hemorrage. J Perinat Med 2001; 29: 351-356.

Recebido em 01 de dezembro de 2003

Versão final apresentada em 26 de outubro de 2006

Aprovado em 30 de outubro de 2006 